\title{
Development of an automated in situ fracture stage for a ToF-SIMS system
}

\author{
Arran R. Wood, Nicolas Benedetto, Nigel Hooker, Edward Scullion, \\ Paul A. Smith, John F. Watts
}

\author{
Surrey Materials Institute and Faculty of Engineering \& Physical Sciences, \\ University of Surrey, Guildford, Surrey, GU2 7XH, UK
}

KEYWORDS: ToF-SIMS; In situ fracture; composite materials

\begin{abstract}
The design philosophy and implementation of a UHV, PC controlled, automated in situ fracture stage for a surface analysis system is described. An example ToF-SIMS spectrum is shown to illustrate the improvement in spectral quality obtained from polymer matrix fracture surfaces prepared using this fracture stage compared to those obtained using samples prepared following testing in a conventional tensile testing system. To a degree the system is modular in design as by changing the capacity of the load cell it is possible to reduce or increase maximum loads as the specimen type and material demands. The stage has been designed with instrumental flexibility in mind, being designed around commercial SEM-stub type sample mounts, and can thus be used for AES/SAM and XPS investigations, as well as ToF-SIMS analysis, in the authors' laboratory.
\end{abstract}

\section{INTRODUCTION}

Fibre reinforced polymer composites are used in many areas of scientific and engineering endeavour, and the types of fibres in use are many and varied (e.g. carbon, glass, Kevlar) as are the types of polymeric matrices (e.g. polyester, epoxy, thermoplastic). One critical requirement of any composite material is the effective load transfer from the matrix material to the reinforcement media. To this end it is often necessary to treat the fibre reinforcements in some manner to enhance and obtain the required level of adhesion between the two phases. Such a treatment may be carried out at the time of manufacture (as in the case of the coating of freshly drawn glass fibres with an aqueous based size of prepolymer and adhesion promoter) of further downstream in the manufacturing process (the electrochemical oxidation of carbon fibres, for example). Over the last thirty years or so many groups have investigated the role of such treatments on fibre-matrix adhesion and it has become clear that the forensic analysis of composite failure surfaces by surface analytical methods has much to offer in the correlation of interface chemistry with mechanical performance.

In the analysis of such failure surfaces it is preferable to achieve mechanical failure within the UHV chamber of the spectrometer, to avoid the deposition of adventitious hydrocarbon material from the atmosphere which in turn will compromise the quality of 
the spectra obtained, particularly in the case of ToF-SIMS. The University of Surrey Group has designed several such approaches to in situ fracture of composites over the years. One of the most successful was based on the use of a commercial fracture stage (VG Scientific UK) designed for metallurgical studies which brings about fracture in a three point bend orientation (Izod geometry) of a small rod-like specimen. The ability to control the strain rate was described in a design by Prickett et $a l^{1}$, which although controlling the strain rate in a rudimentary manner had no means of recording the applied load.

In this short communication we describe the design philosophy and implementation of UHV, PC controlled fracture stage for a ToF-SIMS instrument, in which the strain rate is under PC control and the load applied recorded using a data logger. In this manner it is possible to record a load vs. extension curve in the preparation chamber of the surface analysis system.

A ToF-SIMS spectrum is presented for a polyester resin system, often used as a matrix material for manufacture of GFRP composites. The benefits of in situ fracture for the surface analysis will be demonstrated for this simple single phase material. Ultimately this methodology will be used to investigate more complex two and three phase composite materials.

\section{EXPERIMENTAL DEVELOPMENT}

The fracture of samples in a UHV environment has been previously conducted using custom built and commercial fracture stages ${ }^{1,2}$. These designs utilised either a hand screw linear drive, or a spring loaded impacter to fracture specimens in the instrument preparation chamber or entry lock. However, there has never been any attempt to automate such systems and thereby enable meaningful mechanical data to be determined as the material is fractured, to compliment the contaminant free fracture surface. In order to achieve this, such a system would require modification to include a load cell and an automated linear drive, in effect replicating the design of a quasi-static tensile test machine. These components should work within the confines of a small preparation chamber, operate in UHV, be vacuum compatible and mount to standard Conflat ${ }^{\mathrm{TM}}$ flanges found on most surface analysis instruments.

The preparation chamber of the TOF.SIMS 5 instrument (ION TOF GmbH) has two vertically aligned ports designated for such a fracture stage. An initial design was proposed, as shown in Figure 1, and a systematic approach taken for construction. A four phase, single axis, stepper motor controlled linear drive with controller (Vacuum Generators, UK) with $25 \mathrm{~mm}$ of travel was selected. The stepper motor was connected to a PC and controlled via McTerminal software (McLennan, UK).

In order to measure the load a $25 \mathrm{lbs}(111 \mathrm{~N})$ rated tension/compression load cell (Model 41, RDP, UK) was selected which was interfaced to strain indicator recorder unit (P3, Vishay, UK). 
To validate the automated rig a test platform was designed which replicated the preparation chamber geometry. The linear drive was bolted to the test platform using Conflat $^{\mathrm{TM}}$ flanges. The load cell however required modification in order to make it vacuum compatible and attach to the preparation chamber. In order to mate with the preparation chamber Conflat ${ }^{\mathrm{TM}}$ flange the load cell was sandwiched between a closing flange and a matching flange using Viton ' $\mathrm{O}$ ' rings. The matching flange could then be fitted to the preparation chamber Conflat ${ }^{\mathrm{TM}}$ flange. The closing flange had a recess machined in it to allow for movement of the load cell's lower diaphragm and expose it to region of vacuum. The lower arm of the load string was designed with a hollow tube section and a small aperture serving as a vacuum equilibriator, meaning that both load cell diaphragms experience equal vacuum therefore load variations are caused purely by the straining in the load string, not by vacuum variation. A schematic diagram of the load cell design is shown in Figures 2.

The load cell and strain indicator recorder were connected and firstly checked using standard weights, next the whole fracture stage was validated by calculating the spring constant of a known spring. The fracture stage was then used on the test platform to perform micro-compact tension tests utilising specially designed stubs ${ }^{1}$ at atmosphere for various samples and compared to a body of data measured on a quasi-static tensile test machine.

Once the automation was validated the load cell and linear drive were transferred from the test platform and fixed to the ToF-SIMS preparation chamber where vacuum integrity checks were performed on each component. The installed fracture stage is shown in Figure 3. The specimen mounts were those designed and manufactured by Prickett et al to achieve Mode 1 loading for the earlier fracture stage. The use of such mounts, based on the VG Scientific ESCALAB MkII stub, ensures maximum flexibility of the fracture stage as it is readily assembled on our ESCALAB MkII or Thermo Scientific MICROLAB 350 scanning Auger microscope.

Once installed onto the preparation chamber the in situ fracture stage was used to test a micro-compact tension specimen in UHV. A plaque of unsaturated polyester resin, supplied without the addition of the silica thixotropic agent, (Crystic 2-406 PA, Scott Bader) was manufactured according to the manufacturers instructions. Micro-compact tension specimens were fashioned in accordance with ASTM D5045, with $\mathrm{W}=12 \mathrm{~mm}$.

The sample was pre-cracked as normal by tapping a fresh razor blade into a sawed notch. The specimen was then mounted in the grips and manually loaded through the wobble stick port of the preparation chamber and seated in the lower load string arm. The upper load string arm (equivalent to the crosshead of a quasi-static test machine) was then lowered onto the upper grip and a pin manually inserted to lock the specimen in place. The wobble stick was then replaced and the lower half of the specimen pinned into the lower load string arm using the prong attached to the wobble stick. The preparation chamber was then pumped to UHV, typically reaching $5 \times 10^{-7}$ mbar. 
The strain indicator recorder was set to record at one second intervals and the linear drive started, running at a constant velocity of four steps per second, equivalent to $2.5 \times 10^{-6} \mathrm{~m}$ $\mathrm{s}^{-1}$. The force was then recorded as the specimen was strained to failure. An example of the test being conducted is shown in Figure 4. Upon failure of the specimen the linear drive was fully retracted. The prong pinning the lower half of the specimen was removed. Using the wobble stick, the lower half of the specimen was removed from the load string, mounted in the sample platen and transferred to the analysis chamber to perform ToFSIMS analysis of the fracture surface without exposure to atmosphere.

\section{RESULTS AND DISCUSSION Mechanical data}

The recorded force vs. displacement plot for the resin micro-compact tension specimen tested in UHV conditions, Figure 5, was analysed and the critical stress intensity factor, $\mathrm{K}_{\mathrm{IC}}$, determined as described in ASTM D5045. $\left(\mathrm{G}_{\mathrm{IC}}\right.$ values could not be calculated as the specimen strain could not be measured) The measured $\mathrm{K}_{\mathrm{IC}}$ value was $0.48 \mathrm{MPa} \mathrm{m}{ }^{1 / 2}$, slightly lower than previously determined values ${ }^{3}$ for $\mathrm{K}_{\mathrm{IC}}$ using macro-CT specimens, $0.64 \mathrm{MPa} \mathrm{m}{ }^{1 / 2}$. A more extensive testing scheme will be conducted in future to verify these data.

\section{Surface Analysis}

ToF-SIMS analysis was performed on the micro-compact tension fracture surface generated in situ using a TOF.SIMS 5 (ION-TOF GmbH), fitted with a Bi liquid metal ion source. Polyatomic $\mathrm{Bi}_{3}{ }^{+}$was used as the primary ion source and the bunched mode selected to obtain spectra with high mass resolution and sensitivity over a $200 \times 200 \mu \mathrm{m}$ area using a resolution of $128 \times 128$. The positive spectrum can be seen in Figure 6a). Characteristic peaks associated with fragment ions of the polyester resin have been identified and agree with a previous study of the same material ${ }^{3}$. The probable origin and chemical structure of these characteristic peaks is presented in Table 1.

Careful observation of the spectrum revealed some interesting features. Typically one would expect to observe a relatively strong sodium peak at $23 \mathrm{u}$ associated with contamination from the atmosphere, however there is very little sodium present in the spectrum. Similarly there are no peaks at $73 \mathrm{u}$ or $147 \mathrm{u}$, attributed to poly(dimethylsiloxane) (PDMS) a very mobile surface contaminant present in the atmosphere.

Normally organic fragments of the form $\mathrm{C}_{\mathrm{x}} \mathrm{H}_{2 \mathrm{x} \pm 1}$ follow a standard pattern in ToF-SIMS spectra where the peak intensity increases the higher saturated the carbon chain becomes. However this is not the case for this spectrum. Magnifying a segment between $50-60 \mathrm{u}$, Figure $6 \mathrm{~b}$, more clearly shows that the counter is true, the intensity is higher for the unsaturated end of the carbon chain.

For a positive spectrum of a polymeric material prepared in atmosphere at a nominal mass, in the low mass range the hydrocarbon peak intensity would normally dominate the 
oxygen containing peak. However as Figure $6 \mathrm{c}$ shows, the peak at $55 \mathrm{u}$, the opposite is true for the sample prepared in UHV, counter to normal observations. The causes of these observed phenomena will be investigated in future studies.

\section{FUTURE WORK}

The fracture stage will be used for a full and thorough investigation of the effect of fracture in UHV to the fracture surface chemistry. Further tests are planned to validate the mechanical data. The fracture stage design permits a range of different tests specimens to be fractured. The limiting factors being the maximum displacement of the linear drive, $25 \mathrm{~mm}$, the small confines of the preparation chamber and the load limit of the load cell $25 \mathrm{lbs}(111 \mathrm{~N})$. However the modular nature of the design permits a higher rated load cell to be interchanged enabling higher load capacity, extending the range of sample types and materials available to be tested. It is anticipated that further developments will permit sample loading in UHV, thereby speeding up investigations and enable the second fracture surface to be manipulated and analysed also.

\section{CONCLUDING REMARKS}

An automated in situ fracture stage has been developed to enable mechanical testing of materials in UHV conditions to reduce contamination and improve spectral failure analysis. $\mathrm{K}_{\mathrm{IC}}$ data has been determined and ToF-SIMS analysis performed on a polyester fracture surface without exposure to atmosphere.

\section{ACKNOWLEGEMENTS}

ARW wishes to thank Dr Marie-Laure Abel and Dr Steve Hinder for assistance with ToF-SIMS analysis and interpretation.

\section{REFERENCES}

1. Prickett AC, Smith PA, Watts JF. Surf. Interface Anal. 2001; 31: 11-17

2. Vickers PE. PhD Thesis, University of Surrey, 1998

3. Jesson DA. PhD Thesis, University of Surrey, 2005

4. The Static SIMS Library, Part 2 - Organic Materials, SurfaceSpectra Ltd., Manchester, UK, Wiley, 1999

5. The Münster High Mass Resolution Static SIMS Library, Edition V1.3, Vol. II Polymers

6. Static SIMS Handbook of Polymer Analysis, Perkin-Elmer Corporation 


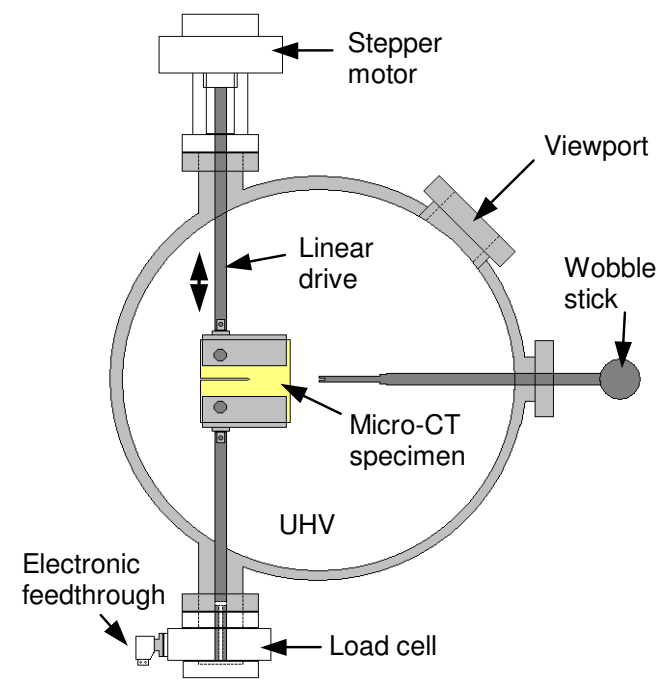

Figure 1. Schematic of in situ fracture stage design concept

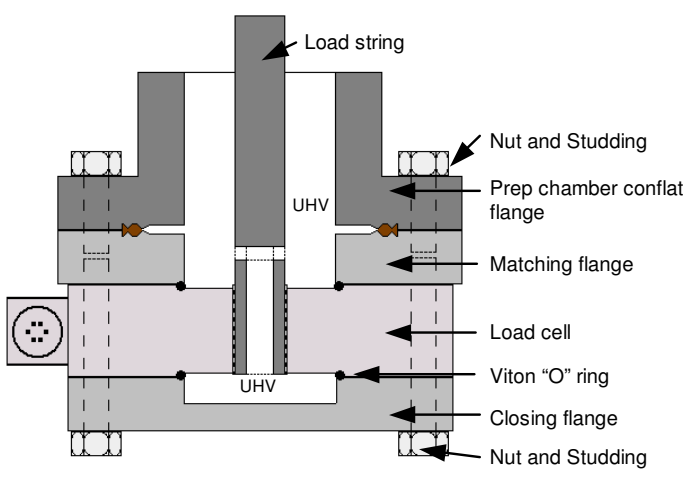

Figure 2. Schematic of load cell assembly

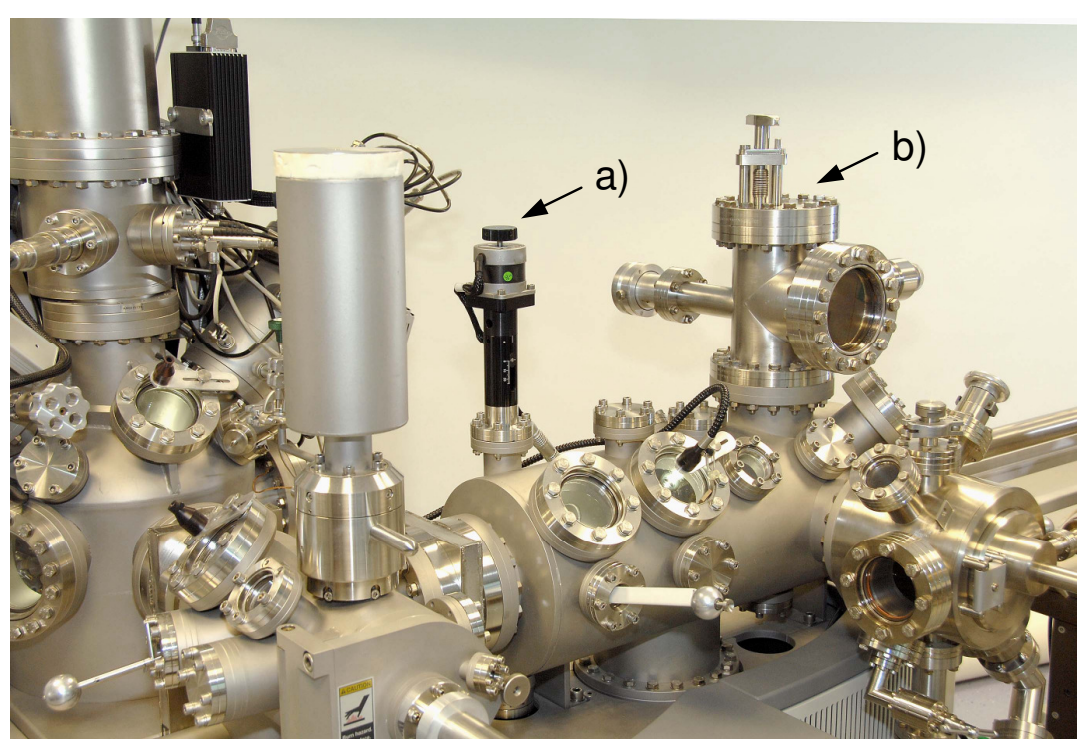

Figure 3. ToF.SIMS 5 preparation chamber in situ fracture stages; a) quasi-static fracture stage, b) impact fracture stage 


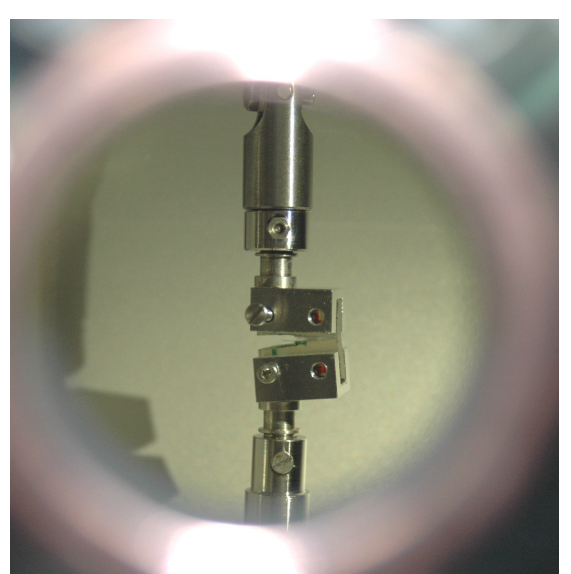

Figure 4. Micro-compact tension specimen being tested in situ

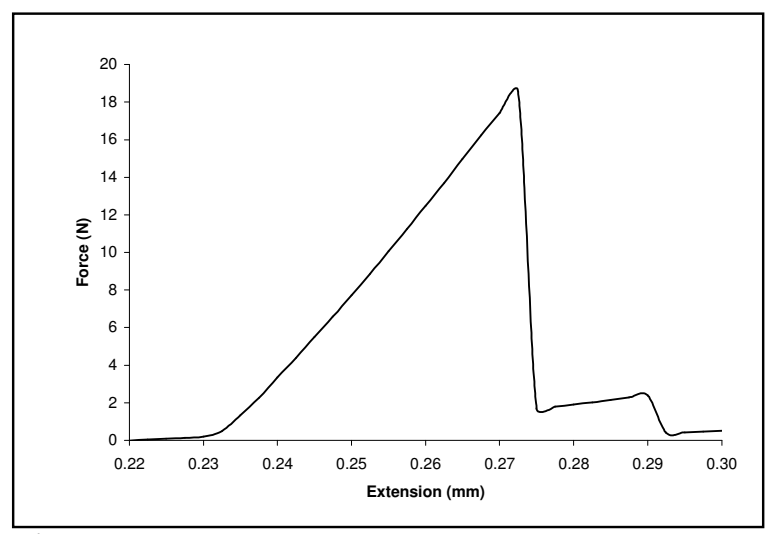

Figure 5. Force vs. extension plot of polyester resin micro-compact tension specimen tested in UHV 

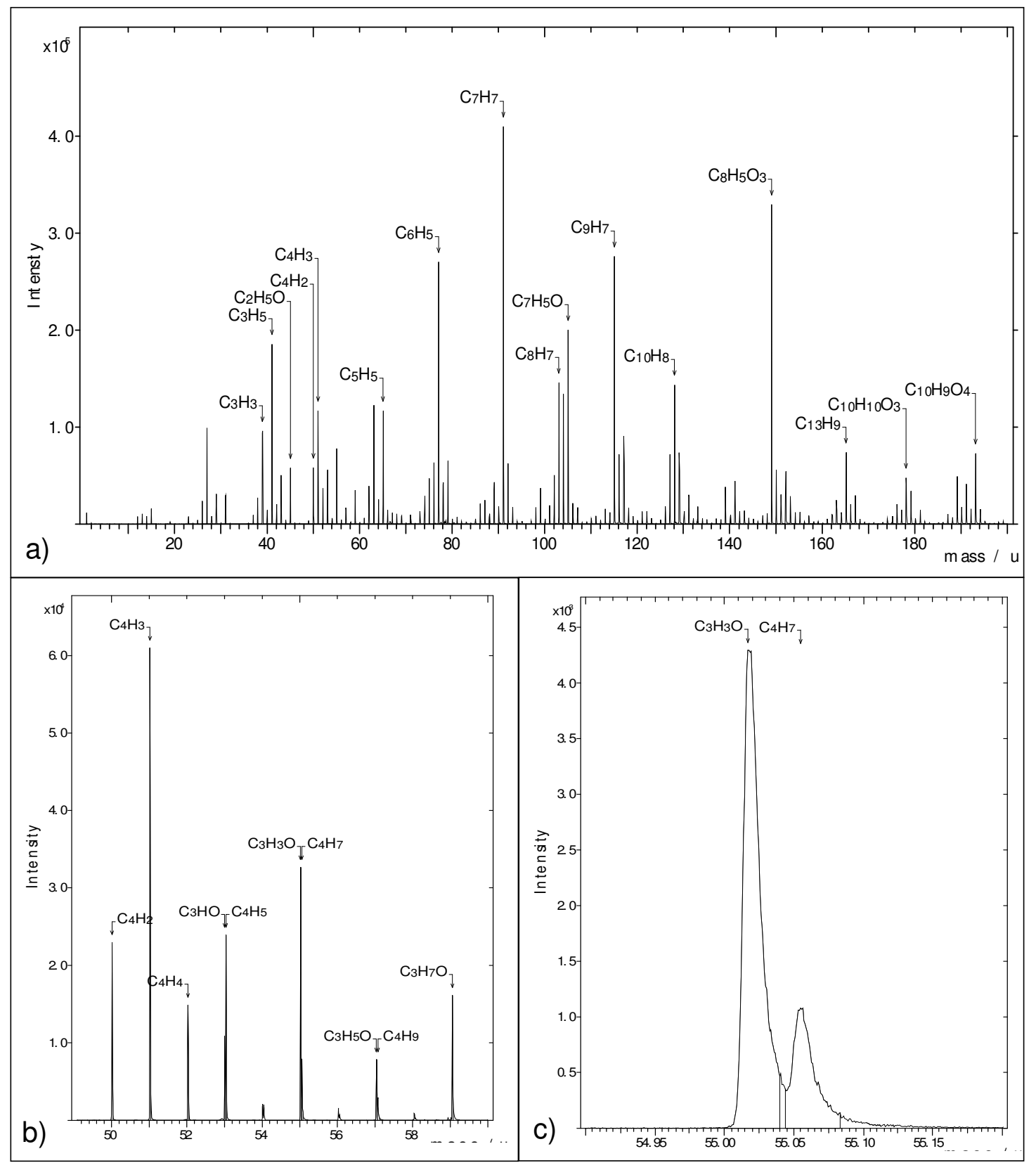

Figure 6. Positive ToF-SIMS spectrum of polyester resin micro-compact tension fracture surface generated in UHV; a) mass range $0-200 \mathrm{u}$, b) magnified view between mass range $50-60 \mathrm{u}, \mathbf{c}$ ) peaks at nominal mass $55 \mathrm{u}$

Table 1. Polyester characteristic peaks ${ }^{3-6} ; \mathrm{G}$ (glycol), MA (maleic anhydride), TP (terephthalate)

\begin{tabular}{|c|c|c|c|}
\hline Nominal mass $(\mathrm{u})$ & Fragment ion & Structure & Origin \\
\hline 39 & $\mathrm{C}_{3} \mathrm{H}_{3}^{+}$ & $\triangle^{+-}$ & $\mathrm{TP}$ \\
\hline 41 & $\mathrm{C}_{3} \mathrm{H}_{5}^{+}$ & $\mathrm{H}_{2} \mathrm{C}=\mathrm{CH}-\mathrm{CH}_{2}^{+}$ & $\mathrm{G}$ \\
\hline 45 & $\mathrm{C}_{2} \mathrm{H}_{5} \mathrm{O}^{+}$ & ${ }^{+} \mathrm{CH}_{2}-\mathrm{CH}_{2}-\mathrm{OH}$ & $\mathrm{G}$ \\
\hline
\end{tabular}




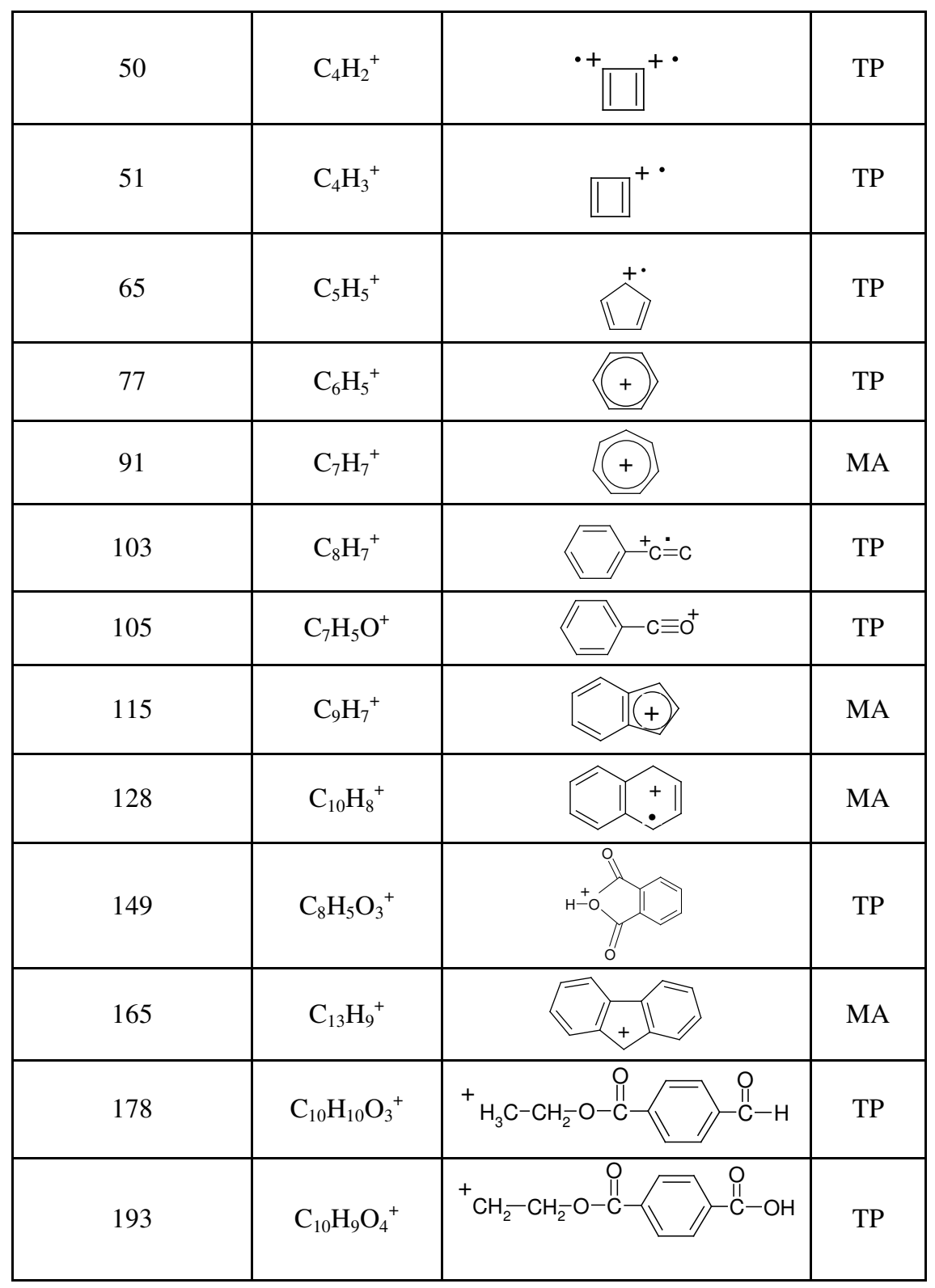

